\title{
TB, or not TB?
}

\author{
Michael A. Valentino, MD, PhD
}

The patient is a 43-year-old African American male with a past medical history of hypertension, hyperlipidemia, and chronic low back pain who presented to the ER for worsening dyspnea and a 30-lb weight loss over the past 3 months. He also reported a nonproductive cough over this period of time as well as an eruption of small $1 \mathrm{~cm}$ flesh-colored nodules on his extremities and trunk. He denied any fevers, chills, or night sweats. In the ER, he had a chest x-ray performed (Figure 1) which showed diffuse centrilobular nodules visualized throughout the lung fields in a miliary pattern. He subsequently had a CT Thorax (Figure 2) performed which showed innumerable miliary nodules and extensive mediastinal and hilar lymphadenopathy. There was suspicion for miliary tuberculosis (TB), and the patient was admitted and placed on airborne precautions. He was also found to be hypercalcemic with calcium of $11.9 \mathrm{mg} / \mathrm{dL}$ (normal range $8.5-10.2 \mathrm{mg} /$ $\mathrm{dL}$ ) on admission, which improved with IV fluids.

The patient was incarcerated over 10 years ago but had no other risk factors for tuberculosis: no travel to endemic areas, no history of homelessness, no substance abuse, and no known exposure to an individual with TB. Consultation by Infectious Disease, Pulmonary, and Dermatology was requested. The differential diagnosis included: TB, disseminated fungal infection, and sarcoidosis. A Quantiferon TB Gold test and three daily acid-fast bacilli sputum cultures were negative. He also had negative tests for cryptococcal antigen, histoplasma antigen, and blastomyces antibody. He did, however, have an elevated serum level of angiotensin-converting enzyme at $440 \mathrm{U} / \mathrm{L}$ (normal range 9-67 U/L). Dermatologist biopsied one of the patient's cutaneous nodules, and the pathology revealed sarcoidal granulomatous dermatitis. Thus, the patient was diagnosed with disseminated sarcoidosis and initiated on steroids. The patient's hypercalcemia was also due to sarcoidosis, as he had a low intact parathyroid hormone level and an elevated calcitriol level consistent with hypercalcemia of granulomatous disease. With treatment with steroids, the patient's dyspnea improved. He was discharged on oral prednisone and recommended to follow-up with Pulmonary.

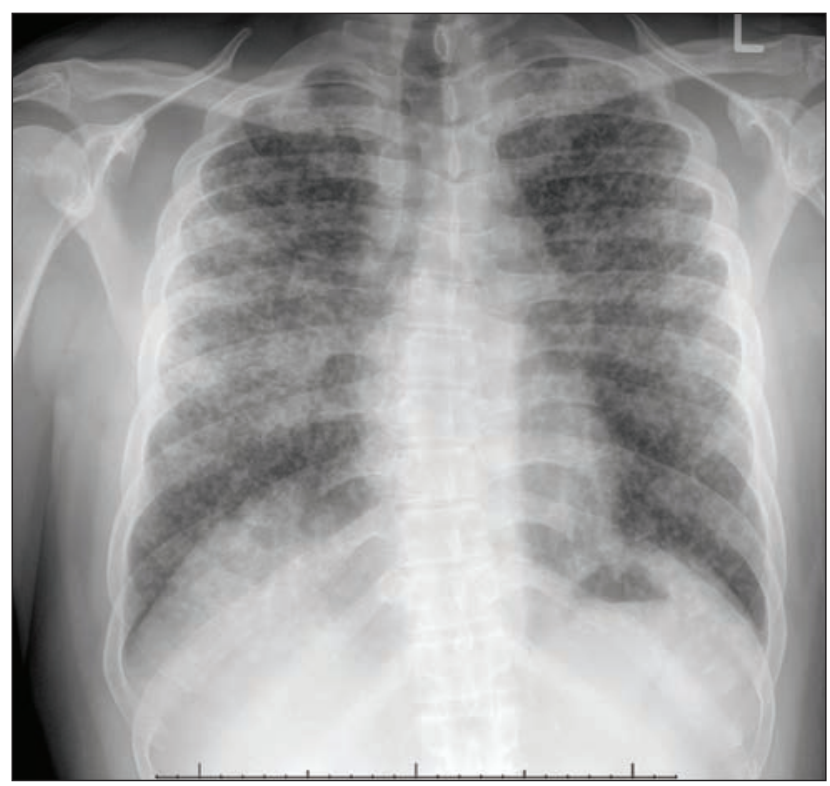

Figure 1: Admission chest X-ray

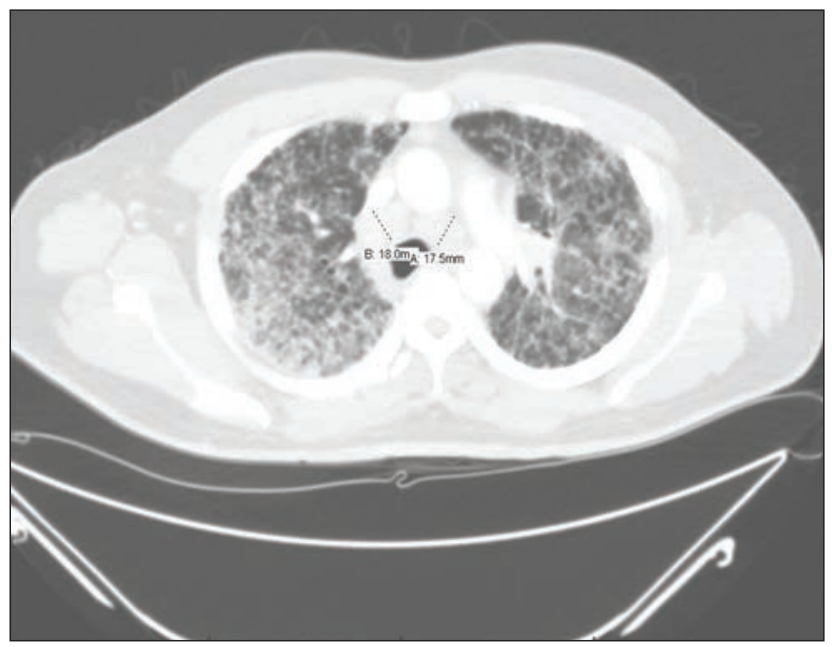

Figure 2: Admission CT Thorax. 